\title{
La matrice ou le drageoir aux transgressions Lectures croisées des pathologies féminines fin-de-siècle
}

\author{
Laure-Hélène TRON-YMOMET \\ Université Lyon 3
}

Dans son terrible essai sur La Femme, Michelet finit par postuler que « l'homme est un cerveau, la femme une matrice » (cité par Borie 158). L'auteur rappelle par cette sentence la force d'une pensée essentialiste où « la femme » serait non seulement définie par des caractéristiques précises et immuables ${ }^{1}$ mais aussi et surtout par une profonde aliénation à son sexe. La croyance n'est pas neuve et ses antiques fondements ne cessent de se réactualiser au siècle charcotien. Les Goncourt décrivent ainsi la femme réglée comme un «animal fou, méchant, trouvant un âpre plaisir aux souffrances de ce qui lui est associé dans la vie » (Goncourt 669) quand Huysmans fait d'elle le « lieu de la plus haute vulgarité » (Huysmans 58). Son tempérament aussi bien que son corps dégoûtent (Dorlin 9). Ce dernier n'est-il d'ailleurs pas toujours malade et incapable de santé puisqu'il saigne incoerciblement chaque mois (Rouch 126) ? Toute définition de la féminité semble se réaliser à l'aune d'un retour à l'organique ou, plus précisément encore, à ce que nous appellerons ici matrice : pris dans une acception plus étendue et symbolique, le terme ne désigne plus seulement l'appareil reproducteur mais aussi ce qui le constitue. Vagin, utérus, ovaires, fibres, sensations deviennent ainsi gouffres de perdition, drageoirs aux transgressions.

L'expression «drageoir aux » réfère immédiatement à Huysmans ami de Charcot - et à son Drageoir aux épices paru en 1874. Si cet hapax poétique huysmansien n'est pas au centre de nos préoccupations, force est de constater qu'un tel syntagme permet de développer une large imagerie : le drageoir, objet d'orfèvre, contient à la fois le plaisir (la dragée sucrée) et le transgressif (il n'est pas bon de céder à ses désirs, surtout en ces jours où le healthy way of life s'impose ${ }^{2}$ ). L'ouvrir reviendrait alors à une sorte d'acte de folie. La référence à Pandore, bien que présente, se retrouve cependant inversée puisqu'il revient à l'homme d'ouvrir la boîte et d'y trouver la femme ou, plus particulièrement, son sexe. La matrice porte en elle cette dualité

\footnotetext{
1 Tandis qu'il n'en va pas de même pour «l'homme », ce que rappelle Thomas Laqueur : "Woman alone seems to have "gender" since the category itself is defined as that aspect of social relations based on difference between sexes in which the standard has always been man» (Laqueur Making Sex 22).

${ }^{2}$ Il n'y a qu'à regarder le succès des comptes Instagram et des livres qui portent sur les régimes, la nourriture équilibrée ou les recettes vegan. En 2017, le célèbre New York Times a créé un espace spécialement dédié aux livres «healthy » sur son site, avec critiques littéraires et culinaires <https://www.nytimes.com/books/best-sellers/health/>.
} 
profonde qui était l'apanage du coffret : objet désiré et désirant, elle ne cesse de renvoyer à l'expérience d'une permanente transgression.

En effet, depuis Hippocrate, l'organe génital féminin est marqué du sceau de la passivité et de la déviance puisqu'il n'est qu'un renversement des attributs masculins. Ce postulat traverse les siècles et semble trouver une sorte d'acmé à la fin du XIX ${ }^{\mathrm{e}}$ siècle. Dans son ouvrage consacré aux personnages féminins chez les Goncourt, Domenica De Falco réinscrit la misogynie des frères au cœur d'une société qui place la femme "d'emblée dans une situation de faiblesse. Sa constitution en est la preuve qui se présente plus proche de celle de l'adolescent que celle de l'homme » (De Falco 26). À la réduction physique, répond celle sexuelle. Et, alors que la fin du $\mathrm{XIX}^{\mathrm{e}}$ siècle représente un moment de bascule en ce que la bourgeoisie se dégoûte progressivement de la fécondité (Berthiaud 94), un tel réarrangement des mœurs tend à créer un nouveau paradoxe : ce n'est pas sous prétexte qu'elles ne doivent plus faire d'enfants que les femmes peuvent pour autant concourir à la liberté sexuelle. Le premier argument est bien entendu scientifique et se fonde sur la physiologie puisque "l'œuf ne serait qu'une cellule inférieure, ayant un rôle passif, fonctionnant comme un simple réceptacle du spermatozoïde qui est, lui, susceptible d'un développement supérieur et possède un rôle actif dans la fécondation » (De Falco 26). L'envie de ce sexe passif ne serait ainsi stimulée que par un besoin de satisfaire l'homme, la bonne tenue du foyer définissant encore la passion organique. Le psychiatre Krafft-Ebing s'appuiera sur les travaux médicaux et reprendra cette théorie dès l'introduction de sa Psychopathia sexualis, mettant au jour une pensée alors restrictive de la sexualité de la femme ${ }^{3}$ : enfin libérée du joug de l'enfant, elle peut maintenant dormir en paix (Krafft-Ebing 34). L'affirmation scelle une approche déterministe de la constitution féminine, corps toujours souffrant qu'il faut à tout prix discipliner.

La fin du XIX ${ }^{\mathrm{e}}$ siècle est également marquée par la naissance d'une complémentarité fondamentale entre la médecine et la littérature, la naissance du naturalisme entérinant un nouveau paradigme herméneutique. Les progrès fulgurants de la science à partir de 1850 entraînent une émulation féconde du côté littéraire et, a fortiori, du roman, genre le plus apte à composer avec le prosaïsme du monde. Déjà séduits par les ambitions réalistes, les romanciers de la seconde moitié du siècle cherchent à penser avec et pour la médecine, alors terrain privilégié d'exploitation. Science en train de se bâtir, la médecine oscille entre les anciennes théories vitalistes et une nouvelle pensée organiciste qui fragmente le corps en différents sièges (Cabanès 158). À chaque organe correspond une ou des pathologies dont les découvertes se multiplient. Le corps se mue alors en palimpseste que le praticien ou le romancier, explorateurs d'un nouveau genre, dévoilent et déchiffrent. Plus encore, en l'espace corporel continue de se lire un rapport métaphorique qui le fait accéder au statut de

\footnotetext{
${ }^{3}$ Nous utiliserons l'expression « la femme » par praticité et non par adhésion à l'essentialisme.
} 
symbole sociétal. Jean-Louis Cabanès explique ainsi que «dans la littérature dite réaliste ou naturaliste, les thèmes physiologiques ou pathologiques s'imposent de manière quasi obsédante comme figure privilégiée de toutes les déviances, du désordre social, voire de la finitude humaine »(Le Corps 12 ). Le corps rejoue sa prime polysémie : la comparaison entre organisation sociale et organisme vivant, rejetée par le critique, semble pourtant avoir toute son importance. Ainsi, forts d'une lecture des corps et des chairs, médecins et écrivains choisissent d'incarner le sentiment apocalyptique qui s'empare du peuple français à l'arrivée de 1900 dans l'organe génital féminin. Celui-ci acquiert en effet le statut de nouvel objet scientifique dès le début du siècle, la première opération du péritoine, réalisée en 1908, exposant aux yeux de tous un organe qui était jusqu'alors inaccessible. Dès lors, les textes fondateurs se multiplient et les médecins ne cessent de présenter à leurs pairs les résultats de leurs recherches matricielles. La force objective ne semble cependant pas l'apanage d'une gynécologie qui reste fondamentalement militante (Borie 164) : règles, accouchements, détraquements s'observent sous la loupe d'une certaine morale. Les médecins, alors qu'ils dénigrent de plus en plus la parole religieuse, chercheraient à conquérir corps et âmes, la matrice apparaissant comme objet à la fois transgressif (on ose en parler) et fondateur d'une nouvelle pensée séculaire. À leur suite, les romanciers vont tenter de dire et de représenter ce nouvel espace sémantiquement vacant. Leur but n'est-il pas similaire ? Leur peinture agit-elle selon un simple principe de dévoilement ? En réalité non, puisque, nous l'avons dit, la matrice complexifie le regard clinique, doublant la relation symptômes-maladies d'une dualité pathologie-société. De fait, le sexe féminin révèlerait l'inscription de toutes les transgressions et se ferait compendium des maux du siècle. Son dévoilement signerait alors l'avènement d'une révélation néfaste. À la manière d'Èves modernes, si les femmes se libèrent sexuellement, la désolation s'abat sur l'humanité.

Mais quelles sont ces transgressions ? Si leur action est néfaste, pourquoi les dévoiler ? La question de la finalité reste prégnante : pourquoi exposer le mal ? Faire le choix d'une telle thématique n'est-ce pas proprement provocateur? Ne traduit-il pas, in fine, une volonté du romancier et du médecin de s'affirmer soi-même comme transgressif ? Dans quelle mesure cette exposition ne correspondrait-elle pas à un exercice cathartique ?

Dans le sillon des nombreuses recherches philosophiques, historiques et sociologiques menées sur la relation «science et genre » (Elsa Dorlin, Evely Fox Keller, Emily Martin, Joelle Wiels par exemple), notre étude, beaucoup plus modeste dans ses ambitions, explorera ces questionnements sous l'angle de la littérature fin-de-siècle. Ainsi, dans premier temps, nous dresserons une petite taxinomie - non exhaustive - des transgressions sexuelles liées à la matrice puis, dans un second temps, nous exploreront les déviances cliniques 
qui voient le jour à partir de 1850. Ce travail sera conduit par une ambition comparatiste, où résonneront textes cliniques et littéraires.

\section{Transgressions sexuelles : exorciser la bête.}

Dans Naissance de la clinique, Foucault rappelle qu'à partir du XIX siècle la médecine tente de s'organiser autour d'une normalité du corps humain (Foucault 36). Naît alors un intérêt fécond pour les corps «déviés » ou transgressifs qui offrent une vérité à révéler sur cet ordre naturel. Alors que la matrice avait toujours donné lieu à des considérations genrées où s'exprimait une certaine inquiétude (Dorlin 9), le discours médical, qui scrute les corps, vient plus que jamais offrir une caution à cette peur, faisant des femmes des êtres profondément pathologiques, donc monstrueux. Les médecins déploient l'imagerie de ces déviances qui s'organisent selon plusieurs catégories: transgressions psychiques, cliniques et sexuelles. Si le triptyque est éloquent, force est pour nous de le restreindre en excluant immédiatement hystérie et syphilis du champ d'étude, topiques déjà balayées par la critique (pour l'hystérie voir par exemple Sabine Arnaud, 2014 et Nicole Edelman, 2013 ; pour la syphilis voir par exemple Patrick Wald Lasowski, 1982 et Jean-Louis Cabanès, 1996). Reste à justifier plus amplement cette éviction. Alors que le terme d' «hystérie » réfère immédiatement à l'organe, le siège de cette maladie découverte par Hippocrate et renouvelée par Charcot à la fin du XIX ${ }^{\mathrm{e}}$ siècle est progressivement réévalué, les ovaires ne constituant bientôt plus que la caisse de résonance d'une affliction fondamentalement nerveuse (Arnaud 186). Il n'est ainsi plus affaire de matrice mais de cerveau ; Michelet est renversé.

La syphilis naît dans l'esprit commun d'une contamination fondamentalement féminine : les femmes, et a fortiori les prostituées, se muent régulièrement en relais du mal. C'est sur cette croyance que parie la duchesse d'Arcos de Sierra Léone, dans la nouvelle de Barbey d'Aurevilly, pour ternir le nom de son mari : après le meurtre de son amant, elle quitte son pays natal, devient fille de joie à Paris et s'éteint dans les souffrances de la vérole (Barbey d'Aurevilly, 1883). La mauvaise foi médicale ira alors jusqu'à faire de la mauvaise hygiène des prostituées le vecteur d'une prime contagion (Cabanès Le corps 91). Mais si la destruction de l'homme se réalise toujours au contact des muqueuses matricielles, la maladie s'écrit loin d'elles : ce sont les corps tout entiers qui sont frappés de décomposition. Cette ouverture spatiale contredit quelque peu notre volonté de nous circonscrire à l'organe génital féminin.

Trois transgressions sexuelles s'offrent alors comme objets d'étude pertinents et peu déroulés par l'exégèse littéraire : la nymphomanie, le saphisme et l'onanisme.

\section{Mise en spectacle de la nymphomanie}


Si le XVIII ${ }^{\mathrm{e}}$ siècle sacre la libertine, le $\mathrm{XIX}^{\mathrm{e}}$ siècle tente de l'annihiler et en dénonce les vices. Les nymphomanes procèdent de cette inquiétante étrangeté où le pathologique ne cesse d'affleurer sous la fascination (Chaperon 62). Et c'est justement parce que le sexe féminin, lorsqu'il est mis en prose, est sans cesse transmué en «cas» qu'il conduit, dans son expression, à la complémentarité des écritures littéraires et médicales.

Dans son ouvrage sur La Folie lucide, le neurologue Ulysse Trélat relate une de ses leçons publiques sur la nymphomanie; après un bref exposé théorique, vient le temps de l'expérience pratiquée sur une jeune femme inconnue :

... On eût dit que la vie s'était, en quelque sorte, retirée et accumulée dans les membres abdominaux. Ce qui causa notre surprise dans un accident aussi étrange, c'est que les forces sensitives s'étaient exaltées et, en quelque sorte, concentrées dans l'intérieur de l'organe utérin, au point que la vue seule d'un homme qui serait entré dans la salle de l'hôpital Saint-Louis où elle était couchée suffisait pour déterminer en elle le spasme voluptueux des parties de la génération : toutes les impressions qu'elle éprouvait venaient retentir dans ses organes ; la main de toute personne qui n'était pas de son sexe, posée dans la sienne, elle en avait la sensation dans le vagin. Cette malheureuse avait une telle propension à s'émouvoir, qu'il suffisait de lui toucher un doigt pour y susciter des mouvements contractiles. En parcourant ainsi les diverses parties de son corps, on finissait par agiter toute sa personne, et la monter en convulsions, comme on met en activité les ressorts d'une horloge. Les convulsions duraient près de trente minutes. La malade, pendant ce temps, poussait des gémissements lamentables, et offrait l'image parfaite des convulsionnaires de Saint-Médard. Une pareille situation était vraiment effroyable pour les spectateurs (Trélat 41)

L'auteur, loin d'adopter la prose sèche du protocole scientifique, déploie un style dense où foisonnent les émotions. À une époque où la médecine voudrait se détacher de l'art pour devenir science (Bernard 27), une telle narration a de quoi surprendre. Derrière son ton et sa force stylistique perce un romanesque (Declercq et Murat 8) que nous pouvons étudier comme tel. Ainsi, la mort du corps s'oppose à la vie qui semble habiter l'utérus. La femme est décrite comme une «malheureuse », une « malade » dont l'exaltation des sens est mesurée à l'aune d'un pathétique croissant incarné dans les gémissements : l'expérience sert moins à révéler la démesure du plaisir que celle du pathologique. La femme perd son humanité puisqu'elle est tout entière soumise à son sexe. Le médecin déploie sous nos yeux une symphonie organique au sein de laquelle les tressaillements croissent de manière exponentielle. La fin de l'observation n'est d'ailleurs pas racontée : tout se passe comme si la tension restait palpable de manière infinie. L'enjeu de la représentation est alors clair : il s'agit de faire peur à l'assistance, l'extrait évoquant une extraordinaire hypotypose; nos cheveux se dressent sur nos têtes à la vue d'un spectacle 
« vraiment effroyable ». Reste qu'une certaine naïveté auctoriale perce sous une telle mention, l'auteur dirigeant notre impression vers un but précis, terreur et pitié à nouveau rejouées. Plus qu'en romancier, le médecin apparaît d'abord en prestidigitateur qui expose légitimement aux yeux de tous l'horreur de ses découvertes. L'exposition publique de ce sexe malade n'est pas sans contentement: l'homme domine la nymphomane qui, a priori, devrait le dominer sexuellement. L'expérience se transforme en vengeance médicale, le « cas » devient digne d'un personnage romanesque.

Quelques années plus tard, Lucie Triarche, héroïne de Paul Adam dans Chair molle, incarne à son tour le rôle de la nymphomane mais, cette fois, dans un véritable roman. Après avoir contracté la syphilis avec un client de sa maison close, la jeune femme est renvoyée. Un séjour prolongé à l'hôpital semble la guérir et lui permet de retrouver du travail ainsi qu'un amant. Si son précédent métier avait éteint ses sens, ses entrevues avec un jeune officier lui font connaître ses premiers émois sexuels :

Bientôt la hantise de ces joies, l'attente du moment exquis devinrent ses uniques préoccupations. Elle ne pensait plus qu'à l'amour, saisie d'un étrange malaise, si elle en restait privée quelques temps. Abandon d'elle-même complet, voluptueux, à ce mal.

Et, à minuit, lorsque le concert s'achevait, Lucie dans une grande hâte d'arriver chez elle, prenait la manche de son amant, le forçait à courir pour rentrer plus vite. Dans la chambre, la porte à peine refermée, elle se collait à Charles, elle le serrait, elle l'entraînait sur le divan. Puis le couvrant de son corps, elle mordait ses lèvres goulûment, avait des baisers chauds où leurs langues s'enchevêtraient. Pour apaiser le froid qui la prenait, elle glissait les mains dans les amples manches du dolman. Sous les attouchements fiévreux de l'homme, ses yeux se fermaient, elle renversait la tête, elle se tordait, étirant les jambes, haletait par saccades. Alors la crise devenait plus forte; c'était comme une pesanteur qui roulait dans son ventre, qui montait, laissant après elle un vide délicieux. Du corsage déboutonné en hâte, les seins jaillissaient. Et toujours l'étreinte devenait plus pressante jusque le moment où la fille s'affaissait, la bouche ouverte, les yeux noyés, proférant une plainte rauque (Adam 145-146)

Les échos avec le texte de Trélat sont prégnants, d'autant plus que l'auteur cherche à écrire un roman « à thèse ». Le discours médical peut se lire comme palimpseste de la démonstration : on retrouve « l'étrange malaise » ainsi que l'accroissement perpétuel des spasmes qui aboutissent à la «plainte rauque » d'une femme muée en bête. Les symptômes sont déroulés en même temps que semble disparaître le corps de la jeune femme : tout se concentre dans sa matrice. L'expression quasi oxymorique qui ouvre le passage, « hantise de ces joies », montre ainsi la dualité profonde entre l'état de plaisir suprême et la prescience du pathologique, condamnation dernière. Émergent ainsi des topö̈ d'époque, fondés sur une hyperbolisation du sexe féminin. Et le romancier, à 
l'instar du médecin, appuie à son tour sur la malade, révélant la puissance du pathos à l'œuvre.

Dans la nymphomanie se joue une tragédie organique qui se traduirait en termes freudiens dans le couple éros et thanatos. L'analyse, bien qu'anachronique, sert à révéler le dilemme de la nymphomane et ses attaques sont les symptômes d'un violent tiraillement entre pulsion de vie et pulsion de mort (Freud 254). Or, le sexe de la femme, au XIX ${ }^{\mathrm{e}}$, est appelé à la pulsion de mort puisqu'il doit sans cesse témoigner d'un retour à l'inanimé. Paradoxalement, c'est en ce qu'il est vital que le combat des nymphomanes est transgressif.

Affronter la nymphomanie relève alors du défi pour l'homme civilisé et bourgeois qui affronte sa peur en approchant la matrice contaminée. Sa monstration participe d'un mouvement cathartique qui s'applique aux deux sexes. Dans un chapitre consacré au sexe féminin dans l'espace littéraire fin-desiècle, Mireille Dottin-Orsini postule que « de nombreuses femmes adhérèrent au portrait d'elles-mêmes qu'on leur tendait comme un miroir déformant » (170). Les femmes font basculer les topö̈ littéraires du côté des symptômes et s'effraient d'un mal obsessionnel qui détruit d'un même mouvement le corps et la société. Si une sexualité active les transforme en sujet monstrueux et néfastes, mieux vaut rester objet passif. Minotaure d'un labyrinthe pathologique, la nymphomane se tuerait elle-même avant de tuer les autres, au contraire de la femme saphique, fondamentalement dangereuse pour autrui.

\section{Les héritières de Sappho}

$\mathrm{Si}$, dans le cas de la nymphomanie, médecins et romanciers semblent guidés par un même geste, le saphisme soulève d'autres questions. En effet, lorsque les premiers tentent de corriger cette déviance (Krafft-Ebing, 1886), les seconds l'érigent en tendance tout à fait moderne (Albert, 2005). L'anathème littéraire ne concerne que les pratiques homosexuelles masculines, les femmes profitant d'une aura particulière qui met à mal l'objurgation clinique. Cette dernière s'évertue pourtant à mettre en place un système de remontrances tel qu'elle avait pu le faire pour la nymphomanie : la question préoccupe l'époque et la peur que les femmes ne soient détournées de la procréation par un égoïste érotisme, se lit dans tous les traités hygiénistes et moraux. Dès le XVIII ${ }^{\mathrm{e}}$ siècle, Louis Martineau, médecin chevalier de la légion d'honneur, expliquait que «certaines inflammations, excoriations, ulcérations, contusions vulvaires et clitoridiennes, certaines inflammations de la glande vulvo-vaginale, trouvent leur explication dans le saphisme» (Martineau 4). À mesure que la phrase gonfle, l'énumération fait croître difformités et horreurs jusqu'à s'achever dans le mot diagnostic «saphisme ». Prédicateur profane, le médecin annonce ainsi les plaies qui frapperont les femmes homosexuelles via une terrible - mais succincte - hypotypose matricielle. Son œuvre fait loi et ses successeurs 
n'auront de cesse de rappeler ses prophéties. Au dérèglement nerveux de la nymphomane répondent les distorsions de la tribade. Le monstre reste cependant caché : nous n'avons à ce jour jamais trouvé de témoignage de leçons médicales portant précisément sur l'observation génitale des homosexuelles. Le sujet, s'il n'est pas tabou, est toujours rejeté.

Les romanciers refusent pourtant cette condamnation et n'ont de cesse de réactualiser le mythe saphique. Les Goncourt sont ainsi fascinés par les exploits de leur amie Lagier, avec qui ils causent «tribaderie et pédérastie transcendantales » (Goncourt 1052), Paul Adam octroie à son héroïne, désespérée par les hommes, un «spasme furieux, interminable » lors de son accouplement avec sa coturne (73) quand Zola fait dire à Nana qu'il n'y a « rien de plus commun ni de plus naturel » que le saphisme (Nana 195). Si aucune de ces hérö̈nes ne connaîtra une fin heureuse, leur expérience homosexuelle n'est pas l'unique source de déchéance physique, leur ruine corporelle et morale se réalisant moins par le vice que par l'accumulation des transgressions. Deux hypothèses possibles à ce pas de côté : n'ayant pas accès aux corps de ces femmes, les écrivains ne pourraient juger in vivo des dérèglements engendrés par les pratiques saphiques. À peine énoncé, nous détruisons cet argument : le Comte Muffat de Nana continue malgré tout de voir sa favorite et, s'il est progressivement délaissé, il ne constate jamais de difformité matricielle chez la jeune femme. De même, Paul Adam choisit de décrire très précisément les contractions vaginales que ressent Lucie lors de ses accouplements avec Léa ; à aucun moment il ne sera fait état d'un diagnostic clinique alarmant. Deuxième hypothèse alors : le mythe de Sappho, s'il est a priori scandaleux, se rapporte à la création poétique. Le livre créé par, ou pour, la saphique deviendrait une sorte de substitut à l'engendrement. Dans Sapho, roman de mœurs parisiennes, Fanny Legrand incarne une héroïne à la fois libertine et tragique, dont les amours inspirent un sculpteur, un poète et le romancier lui-même. La salvation se cache dans la production que ce soit celle d'enfants ou de livres.

La déviance n'est alors pas à chercher dans l'acte sexuel mais dans ce qui l'encadre : les femmes ne pourraient échapper à un besoin de rejouer le jeu hétérosexuel, l'une d'entre elle devant nécessairement endosser le rôle de l'homme. Jean Lorrain, dans l'un de ses Portraits de femmes, met ainsi en scène un petit rassemblement de jeunes gens qui sont « hommes de la ceinture aux cheveux, par le veston allant, le pantalon épinglé et le chapeau de feutre ; femmes, de la ceinture aux pieds, par la jupe plissée, les bas de soie et la fine chaussure » (Lorrain 56). La confusion demeure jusqu'à ce que l'une d'elles accouche, l'acte opérant un retour du genre puisque la jeune femme ressemble désormais à la Vierge. Mais les inclinations saphiques de la jeune mère entourent l'événement de drame : sa maîtresse, une «petite blonde ... comme un homme avec des habillements pas chrétiens » tuera le nouveau-né en l'exposant nu aux grands vents qui rugissent à l'extérieur. L'enfant, mâle et de 
surcroît fruit d'une relation hétérosexuelle, dégoûte et devient stigmate de l'autre homme qu'il faut tuer. Les saphiques mettent bien « les hommes en péril de mort en usurpant leur puissance masculine » (Takaï 120). D'ailleurs, Lorrain s'amuse dans le titre de la nouvelle à accoler un rang féminin et un nom mythologique masculin : c'est la «Marquise Hérode » qui détrône l'homme et règne sur le foyer féminin qu'elle a fondé. Bien que Lorrain échappe à l'entrée naturaliste, un tel détour peut se justifier en ce qu'il est l'un des auteurs à avoir le plus parfaitement illustré le concept de « féminilité » fondé par les Goncourt et relayé par Huysmans dans À Rebours. Le terme, mot-valise d'un autre temps, fait jaillir la dimension monstrueuse que l'on croyait omise par les romanciers. Le croisement des sexes - genrés — n'est pas sans risque, surtout lorsque la femme tente de remplacer voire de supplanter l'homme. Dans sa thèse sur Le Monstre, le singe et le foetus, Evanghelia Stead démontre à cet égard que l'imaginaire fin-de-siècle dit l'hybridité féminine en termes de monstruosité, le regard masculin accusant sans cesse le « caractère bestial » des corps féminins dénaturés (Stead 104). Ces derniers constituent les premiers fondements de la tératogonie finiséculaire, tératogonie certes enrichie par les décadents mais déjà soulevée par les naturalistes, tourmentés par les possibles féminins.

En somme, s'il ne tue pas, le vice saphique contribue à dérégler les mœurs, la fascination n'étant que de courte durée. Le mystère s'épaissit cependant puisque les pratiques ne peuvent être expérimentées par ces hommes délaissés et l'opacité, si elle engendre encore quelques poèmes ici, peut conduire au silence, comme c'est le cas pour l'onanisme.

\section{Découvertes et décompositions de l'onaniste}

Depuis le grand ouvrage de Tissot, l'onanisme est devenu signe précurseur d'un danger terrible: l'auteur, à titre d'exemplum, nous livrait l'histoire d'un paysan à l'agonie, ses « manustupations » ayant eu raison de sa santé morale et physique (Tissot 71-75). Même si quelques années auparavant, le médecin charlatan John Marten mettait déjà en garde «les deux sexes » contre cette pratique pernicieuse (Marten cité par Laqueur Le sexe 15), Tissot sera le premier à traiter de la masturbation féminine. Le vice, adopté par les femmes, parait alors «même avoir plus d'activité dans le sexe, que chez les hommes » (Tissot 83). En effet, celles-ci

Sont plus particulièrement exposées à des accès d'hystérie ou de vapeurs affreux, à des jaunisses incurables, à des crampes cruelles de l'estomac et du dos, à de vives douleurs de nez, à des pertes blanches dont l'âcreté est une source continuelle de douleurs les plus cuisantes; à des chutes, à des ulcérations de la matrice et à toutes les infirmités que ces deux maux entraînent; à des prolongements et à des dartres du clitoris, à des fureurs utérines, qui, leurs enlevant à la fois la pudeur et la raison, les mettent au niveau des brutes les plus 
lascives, jusqu'à ce qu'une mort désespérée les arrache aux douleurs et à l'infamie (84)

Le corps des femmes semble, paradoxalement, plus déréglé que celui des hommes, recelant en lui nombre de pathologies aux effrayants symptômes. La liste déroulée par le médecin n'a pas pour vocation d'être exhaustive mais bien de terroriser les consciences : il s'agit de défaire l'antique croyance qui faisait de la perte séminale le plus grand mal de l'onanisme. Le médecin redit la soumission des femmes à leur organisme et, dans la mesure où celles-ci s'écartent de l'impératif moral qui postule "l'importance de la raison individuelle, de la retenue, de la transparence, de la sensibilité, de l'imagination et de l'éducation » (Laqueur Le sexe 293), elles redoublent la déviance : à l'écart moral répond la distorsion physique.

Alors que le sujet fascine toute la société bourgeoise ${ }^{4}$, les romanciers semblent refuser de le traiter. L'omission recèlerait-elle une culpabilité inavouable ? Si la nymphomanie et le saphisme sont des maux particuliers aux femmes, l'onanisme contient un «naturel» abominable commun à tous. Les hommes, soumis à ce même vice, ne se risqueraient pas à dévoiler un sujet qui les amènerait à affronter leurs propres pathologies. La médecine, parce que science a priori neutre et objective, récupère alors la pratique et s'occupe d'en faire le portrait. Cependant, des médecins, tels que Thésée Pouillet, gynécologue français de la fin-de-siècle, abandonnent progressivement dans leurs traités le lexique clinique au profit d'un pittoresque qui permet à l'esprit commun de matérialiser les risques encourus. Pouillet raconte ainsi une série de consultations atypiques qu'il glana tout au long de ses recherches :

Une femme vint consulter Dupuytren ... pour une incommodité qu'elle disait sentir dans le conduit vulvo-utérin. Le toucher fit aisément reconnaître qu'il y avait dans cette partie un corps étranger dont on ne put d'abord déterminer la nature, la malade s'obstinait toujours à ne fournir aucun renseignement à ce sujet. Cependant, à force d'exploration, on parvint à reconnaître que ce corps présentait une large ouverture et une cavité profonde. ... enfin, on réussit [à l'extraire] et on put connaître l'objet mystérieux de tant d'efforts : c'était...quoi ?... un pot à pommade qui avait été introduit par son fond, et au sujet duquel la malade balbutia plusieurs fables qui ne pouvaient avoir aucun crédit (Pouillet 33).

Situation d'énonciation, élément perturbateur, péripéties, mise en suspens grâce à la formule quasi magique «c'était...quoi... ? », l'écriture médicale se transforme en récit au sein duquel perce le subjectif et le burlesque. Tout est mis en place pour mettre à distance : la femme qui lira Pouillet -

\footnotetext{
${ }^{4}$ En témoigne le succès de librairie que connaît le traité de Tissot. Thomas Laqueur le rappelle en ouverture de son livre sur Le Sexe en solitaire (Laqueur 9).
} 
succès de librairie - ne peut s'empêcher de redouter une telle honte. L'homme, quant à lui, poursuit sa destruction méthodique d'une matrice active. La dimension comique oblitère quelque peu la gravité de Tissot mais, si l'issue n'est pas funeste, mort et hystérie constituent encore les grandes conséquences de ces «pollutions matricielles».

Face à de ces représentations, reste à percer l'enjeu d'une telle condamnation. Lors de la création de la femme (Genèse 2.23), celle-ci est nommée en hébreu ishsha quand l'homme est appelé ish : la proximité lexicale souligne, d'un même mouvement, la similitude de l'homme et de la femme mais aussi l'existence d'un «supplément féminin ». La femme, bien qu'elle soit créée de et pour l'homme, est porteuse d'une syllabe surnuméraire, la domination lexicale recelant un partage inégal de la sexualité. La femme, dans un mouvement paradoxal, domine l'homme dans ses plaisirs mais y est aussi assujettie : c'est bien elle qui répondra à l'appel du serpent. Or, le moment de la connaissance fige la domination sexuelle d'Ève dans une transgression à sanctionner. Nymphomanes, saphiques et onanistes seraient alors des réactualisations de cette domination sexuelle que les hommes se mettent à redouter. La peur finiséculaire s'incarne dans des figures fantasmatiques prêtes à être détrônées. L'exercice est aussi cathartique que stylistique. La femme à la sexualité active devient une bête indescriptible qu'il est bon d'annihiler. Écrivains et médecins fondent ainsi une mythographie négative apte à dire et incarner le sentiment de déliquescence à l'œuvre en cette fin-de-siècle. Plus encore, ils tentent par l'écriture de s'approprier un espace qu'ils ne maîtrisent pas. En ce siècle de progrès, il est bon de réitérer son autorité, ce que les chirurgiens, véritables empereurs finiséculaires, vont s'appliquer à faire.

\section{Transgressions cliniques : creuser la béance.}

La perte du sentiment de maternité propre à la fin du XIX ${ }^{\mathrm{e}}$ siècle entraînerait les femmes à désirer un acte sexuel absolument stérile. Promesses pour les bourgeois de ne pas scinder un capital durement acquis, pour les travailleurs de ne pas épuiser les maigres ressources dans des bouches affamées, les femmes libérées du joug de l'enfant deviennent symbole de liberté.

La médecine s'empare de ce nouveau besoin et se passionne pour deux opérations à haut risque : l'hystérectomie et l'ovariotomie. Entre 1890 et 1900, plus de cinq cent mille femmes seront châtrées en France (Canu 127). Celles-ci trouvent dans ces opérations des expédients à des maternités contraignantes. Tous les prétextes sont bons pour qu'elles soient pratiquées, un mal de ventre, une bronchite, des crises nerveuses justifiant l'évidement total de l'utérus (Canu, 1897). La bourgeoise oisive et mondaine en quête de nouvelles sensations voluptueuses et menée par un désir insensé de se croire libérée de la 
nature, devient la principale patiente des chirurgiens habiles. C'est elle que peint Zola dans Fécondité, roman des dernières heures où l'on voit Sérafine chercher la jouissance plénière avant d'émarger dans la liste des malades imaginaires. Son opération sera effectuée par le docteur Gaude, praticien aux mains tout aussi divines que son nom :

... Et, naturellement, l'opération fut merveilleuse, un miracle de légèreté adroite, l'organe enlevé, envolé, disparu, comme entre les main subtile d'un escamoteur. Et n'étant pas malade, solide, en pleine force, Sérafine la supporta d'une admirable façon, eut une rapide convalescence, reparue dans le monde triomphante, éclatante de santé, ainsi qu'au retour d'une cure sur les Alpes ou sur les bords de la mer bleue (Zola 204)

À la femme se substitue une Vénus prête à se livrer aux voluptés sans restriction. Zola peint ici l'incandescence d'un corps libéré. Or, à la victoire première, succède l'échec. En effet, si les résultats immédiats sont plutôt concluants, les médecins ne prennent que peu garde aux résultats post-opératoires, souvent désastreux :

... Puis, la lente déchéance avait commencé, une sénilité précoce, dont les symptômes, un à un, se déclaraient. Elle n'était plus femme, il semblait que le sexe, amputé, emportait avec lui tout ce qui faisait sa grâce, sa gloire de femme. Puisqu'elle ne pouvait plus être ni épouse, ni mère, à quoi bon la beauté conquérante des épouses et des mères? Ses cheveux tombèrent, elle vit ses dents jaunir et s'ébranler. Il survint aussi une faiblesse progressive de la vue, tandis que des bourdonnements d'oreille, presque incessants, l'affolaient. Mais ce dont elle s'épouvante le plus, ce fut de cet amaigrissement qui la desséchait, la décharnait, balafrée de rides, la peau dure, jaunie, cassante comme un parchemin. ... Et, des deux mains, elle ouvrit, elle arracha son corsage. Sa gorge, ses épaules apparurent, tout le désastre de sa beauté détruite, tout le deuil effroyable de sa chair, autrefois si chaude, si odorante, si éclatante, aujourd'hui crevassée, vidée, tel qu'un fruit trop mûr qui tombe et se gâte. C'était le saccage de sa nudité secrète, la défaite à jamais de l'amour. Et ses deux mains tremblaient d'une honte enragée, quand elle recouvrit peureusement, cette vieillesse hâtive, ainsi qu'un ulcère immonde, qui l'aurait rongée (Zola 272).

Sérafine dévoile ainsi un corps friable, phénomène provoqué par la brutale dégradation de ses fonctions vitales. Les cellules ne semblent plus se régénérer, les organes s'assèchent au rythme des jours, l'organisme lutte pour bâtir ce qui lui fut soustrait sans raison physiologique précise. Le portrait zolien, en un mouvement paradoxal, construit ce qui est en train de se déconstruire, la restitution ne pouvant cependant aboutir à la reconstruction. La matrice apparaît alors comme organe fondateur de la femme, sorte de socle soutenant un corps morcelé. La vision mécaniste de l'auteur sert ici le pathétique d'une scène aussi violente qu'engagée : la bourgeoise est aux antipodes de l'Evangile naturaliste. 
À l'instar de Paul Adam, Zola nous livre un roman à thèse qui reprend propos et inquiétudes d'une partie du corps médical dont Étienne Canu, médecin des hôpitaux de Paris, se fera le héraut.

En effet, en 1897, le gynécologue lance l'anathème contre la castration féminine et, a fortiori, contre ce que nous pourrions nommer « l'hystérectomie de confort». Il réunit ainsi dans son ouvrage de nombreux témoignages de femmes châtrées n'ayant aucune connaissance des conséquences liées à l'opération. Le médecin s'oppose violemment aux chirurgiens de son temps, qu'il peint en scélérats sans éthique. Canu, en enquêteur acharné, reproduit une conversation de cabinet, rapportée par l'un de ses internes :

Nous nous souviendrons toujours du dialogue suivant qui s'engageait dans un hôpital entre un chirurgien et une jeune femme qu'il avait choisie :

« Je ne peux vous guérir que par une opération. On vous fera une petite incision. Vous n'éprouverez aucune douleur, on vous endormira. Avez-vous des enfants?

- Oui, Monsieur, un garçon (Ou non, suivant le cas).

- En désirez-vous?

- Non, monsieur ; je suis si malheureuse ! ...

- Je vous enlèverai quelque chose, ce qui vous empêchera de devenir enceinte. Et vous ne souffrirez plus.

- Tant mieux. Est-ce que...je sentirai...comme auparavant...

- Oui, oui, je vous comprends. Votre mari vous sera aussi agréable, et vous ne ferez plus d'enfant. Vous resterez continuellement jeune fille ; cela vous va-t-il?

- Oh oui, monsieur, je vous remercie!»

Ces erreurs de M. Lucas-Championnière, répétées de bonne foi, amenaient la victime sous le couteau du sacrificateur (Canu 126).

L'auteur déroule une saynète réaliste qui met en lumière les différents enjeux inhérents à la castration de masse : l'opération est, en effet, pratiquée à partir d'un simple prétexte, le pathétique d'une situation sociale misérable justifiant la prise de décision. L'opération et l'organe désignés ne sont jamais caractérisés, le chirurgien promettant d'enlever un simple «quelque chose ». L'accord est tacite, secret et nommer l'objet reviendrait à en dévoiler son aspect transgressif. Reste que la déliquescence de la femme semble déjà programmée : il s'agit bien de mentir à la patiente en lui promettant une jeunesse infinie. Le chirurgien, quelque peu charlatan, manie avec habileté bistouri et performance langagière, se muant en «sacrificateur » des masses. Il est possible de lire en creux du discours une réécriture quasi faustienne du pacte avec le diable, pacte organique dans ce cas: la jeune femme est d'ailleurs «choisie»par le commanditaire qui assouvi son besoin de violence au travers d'un sacrifice totalement vain. Aucun dieu n'appelle à la réalisation d'une telle machinerie, le chirurgien se laisse contaminer par la fureur de son siècle. La transgression est donc moins du côté des femmes que de celui du praticien qui, se croyant maître absolu de tout, cherche à démontrer ses talents via une opération de grande 
envergure. Dans son ouvrage, Canu propose cinquante-sept observations de femmes castrées involontairement : sous la destruction se cache une révélation, celle de l'homme démiurge qui ne cherche plus à soigner mais à réaliser sa puissance, à assouvir ses pulsions via la matrice.

En somme, l'enjeu du dévoilement de cette transgression est moins celui de fonder une mythographie cathartique que d'amener le lecteur à prendre conscience de la violence d'un siècle qui conduit l'homme à épouser ses pulsions humaines. À travers la castration, le chirurgien s'affirme en tant qu'être fondamentalement supérieur, capable de voir, de guérir, de régir l'horreur. Médecins et romanciers s'allient alors pour offrir au monde le spectacle d'un pacte moderne entre l'opérateur et la femme. Cette dernière épuise cependant le sens du sacrifice : n'ayant rien à expier, remettant sa confiance dans les mains de celui qu'elle ne fait plus homme, la voilà condamnée à un martyr inutile, toujours déjà consommé.

\section{Conclusion}

La seconde moitié du $\mathrm{XIX}^{\mathrm{e}}$ siècle se déroule sur une scène d'un théâtre de boulevard intra-utérin. La réaffirmation d'un « éternel féminin », parfois au seuil de l'ésotérisme, passe par la lecture contemplative et par l'admonestation d'une dysmorphie monstrueuse. Nymphomanie, saphisme et onanisme se lisent à travers des chairs fendues et pendantes que les médecins peinent à soigner. La matrice, nouvel objet médical, terrorise dans sa forme peu clinique puisqu'il est impossible de l'observer in extenso. En 1840, James Marion Sims invente un appareil extraordinaire apte à percer le secret des chairs matricielles, c'est la naissance du speculum dont le nom porte l'ambition scopique. Or, s'il est utile pour déceler les maladies de surface, il ne peut fournir un examen total de la cavité. De fait, la matrice, lorsqu'elle n'est pas ouverte sur la table d'opération, continue de se dérober à l'homme. La pulsion scopique masculine est ainsi toujours déjà battue.

Médecins et romanciers se passent alors la plume pour écrire cet organe insaisissable, devenu topos hégémonique d'une représentation de l'horreur. Loin d'une adoration à l'instar d'un deus absconditus charnel, la création du monde n'enfante plus que les vices et la maladie, devenant une bête qui n'a plus peur de sortir au grand jour. La mise en lumière de ses transgressions ploie ainsi sous le joug de trois enjeux, tous marqués du sceau apocalyptique : il s'agit dans un premier temps de peindre la matrice en monstre agent destructeur de la société. En témoigne la multiplication des articles médicaux sur l'aménorrhée : les femmes, confortées par des diagnostics nerveux, viennent à contrôler leur flux hormonal, action quelque peu malthusienne qui, combinée à celle de la guerre, ferait périr le peuple français. Une telle révélation n'est pas sans conséquence car le lectorat, sous le choc, doit apprendre à pallier ces 
défaillances. Le dévoilement des maux matriciels — partant, sociétaux - se fait épiphanie redoutable, terrible révélation. L'ambition cathartique des écrivains se lit à l'aune d'une écriture qui multiplie les ressorts pathétiques tout en confirmant la puissance de l'homme herméneute. La pulsion scopique à l'œuvre est en réalité conduite par une volonté de réaffirmer pouvoir et domination sur le monde : seuls les médecins et les romanciers sont capables de tout voir, de tout dire. Si cette mise à nue peut être vue comme une transgression de l'ordre moral, reste que leur geste, parce que masculin, devient permis, le poétique préventif sauvant l'organique de l'abomination. Ainsi, ces écrivains se font démiurges des pulsions et, d'un même mouvement, nous passons du speculum au spéculaire. 


\section{Bibliographie}

Adan, Paul. Chair molle. Paris : Auguste Brancard, 1885.

Albert, Nicole G. Saphisme et décadence dans Paris fin-de-siècle. Paris : La Martinière, 2005.

Arnaud, Sabine. L'Invention de l'hystérie au temps des Lumières (1670-1820). Paris : EHESS, 2014.

Aurevilly (d'), Barbey. La Vengeance d'une femme. Paris : Mercure de France, $1951: 285-328$.

Bernard, Claude. Introduction à la médecine expérimentale. Paris : Flammarion, 1984.

Berthiaud, Emmanuelle. «Le vécu de la grossesse aux XVIII ${ }^{\mathrm{e}}$ et $\mathrm{XIX}^{\mathrm{e}}$ siècles en France. » Histoire, médecine et santé 2 (2012) : 93-108.

Borie, Jean. «Une gynécologie passionnée. » Misérable et glorieuse : la femme du XIXe siècle, dirigé par Jean-Paul Aron, Complexe, coll. «Historiques », 1984.

Cabanès, Jean-Louis. Le Corps et la maladie dans les récits réalistes (18561893). Lille : Klincksieck, 1991.

---. «Invention(s) de la syphilis. » Romantisme « Nosographie et décadence » 94. Paris : Sedes, 1996 : 89-109.

Canu, Etienne. Résultats thérapeutiques de la castration chez la femme: conséquences sociales et abus de cette opération. Paris : Ollier-Henry, 1897.

Chaperon, Sylvie. La Médecine du sexe et les femmes, Anthologie des perversions féminines au XIXe siècle. Paris : La Musardine, 2008.

Declercq, Gilles et Michel Murat. Le romanesque. Presses de la Sorbonne Nouvelle, 2004.

De Falco, Domenica. La Femme et les personnages féminins chez les Goncourt. Paris : Honoré Champion, 2012.

Dorlin, Elsa. Au chevet de la Nation, Sexe, Race et Médecine (XVIIe-XVIIIe), thèse sous la direction de Pierre-François Moreau, soutenue à Paris IV en 2004.

Dottin-Orsini, Mireille. Cette femme qu'ils disent fatale. Paris : Grasset, 1993.

Edelman, Nicole. Les Métamorphoses de l'hystérique. Paris : La Découverte, 2013.

Foucault, Michel. Naissance de la clinique.1963. Paris : PUF, 2015.

Freud, Sigmund. «Les deux espèces de pulsions. ». Essais de psychanalyse. Paris : Payot, 1981.

Goncourt, Edmond et Jules, Journal : mémoires de la vie littéraire 2. 1851 1865. 1861 et 1864. Paris : Robert Laffont, coll. « Bouquins », 1989.

Huysmans, Joris-Karl. Le Drageoir aux épices.1874.

Laqueur, Thomas. Making Sex, Body and Gender from the Greeks to Freud. Cambridge (Mass): Harvard University Press, 1990.

---. Le Sexe en solitaire. Traduit de l'anglais par Pierre-Emmanuel Dauzat, Paris : Gallimard, NRF, 2003.

Martineau, Louis. Leçons sur les déformations vulvaires et anales produites par la masturbation, le saphisme, la défloration et la sodomie. Paris : Delahaye, 1886. 
Pouillet, Thésée. Essai médico-philosophique sur les formes, les causes, les signes, les conséquences et le traitement de l'onanisme chez la femme. Paris : A. Delahaye, 1976.

Rouch, Hélène. Les Corps, ces objets encombrants. Contribution à la critique féministe des sciences. Paris : IXe, 2011.

Stead, Evanghélia. Le Monstre, le singe et le fotus. Tératogonie et décadence dans l'Europe fin-de-siècle. Genève : Droz, 2004.

Tissot, Samuel Auguste. L'Onanisme, dissertation sur les maladies produites par la masturbation. Paris : Pigoreau, 1817.

Takaï, Nao. Le Corps féminin nu ou paré dans les récits réalistes de la seconde moitié du XIXe siècle. Flaubert, les Goncourt et Zola. Paris : Honoré Champion, 2013.

Trélat, Ulysse. La folie lucide : étudiée et considérée au point de vue de la famille et de la société. Paris : A. Delahaye, 1861.

Wald Lasowski, Patrick. Syphilis : essai sur la littérature française du XIX siècle. Paris : Gallimard, Les Essais. 1982.

Zola, Emile. Nana. Euvres complètes, 1880. Tome IX. Montréal : Nouveau Monde, 2004.

---. Fécondité. De l'Affaire aux Quatre Evangiles, 1897-1901. Montréal : Nouveau monde, 2008. 